\title{
Numerical study of new techniques drag reduction: application to aerodynamic devices
}

\author{
Amine Agriss * (D), Mohamed Agouzoul (D), Abdeslem Ettaouil, and Abdessamad Mehdari (D) \\ Mohammed V University in Rabat, Mohammadia School of Engineering (EMI), Mechanical and Energetic Engineering Research \\ Team: Modelling and Experimentation ERG2 (ME), Avenue Ibn Sina Agdal, Rabat 10090, Morocco
}

Received: 14 January 2021 / Accepted: 6 August 2021

\begin{abstract}
In the present study, new drag reduction techniques applied to aerodynamic structures have been developed. The test cases have been numerically performed using three simplified models. Simulations have been performed by using the CFD software Ansys fluent. The first case deals with a laminar flow over a flat plate. Drag reduction is obtained by corrugating the shape of the plate. The second case treats a laminar flow over a NACA 0012 airfoil. By the addition of a device fixed on the flow separation point, the drag could be reduced. The last case concerns a turbulent flow over the Ahmed body. Drag reduction is obtained by the perforation of a conduit leading a part of the flow from the front to be injected at the rear of the body.
\end{abstract}

Keywords: Simplified models / drag reduction / flat plate / NACA 0012 / Ahmed body

\section{Introduction}

The environmental risks associated with greenhouse gas emissions push researchers to think about more aerodynamically efficient designs. Aerodynamic efficiency allows a drag reduction on the aerodynamic structures. This aerodynamic drag causes losses in terms of energy and a reduction in efficiency.

Many techniques have been developed in order to reduce drag on the aerodynamic structures. In this context, passive and active flow control methods are of major interest in the drag reduction. The shapes modification or the attachment of additional devices are the conventional passive control techniques and are considered to be inexpensive in comparison with the active ones. Active control methods consist of devices placed on the surface of the aerodynamic systems. These devices require actuators for their operation, which consume power.

For testing the different flow control techniques before using them in reality, simplified models are considered. These models are able to reproduce with great precision physical phenomena which occurs in the real cases. In that sense, flat plates are very useful due to their simplicity and their ability to produce very beneficial results. In addition, airfoils and simplified car models, for example the Ahmed body, are widely employed in the aeronautic and the automotive sectors.

\footnotetext{
* e-mail: amineagriss@research.emi.ac.ma
}

Among the various drag reduction techniques applied on flat plates, the injection of air bubbles near the flat plate surface could reduce its friction drag [1]. The use of riblets on the surface of a flat plate could also reduce drag [2]. The use of the large-eddy break-up device (LEBU) could reduce the global skin friction drag [3]. The use of staggered circular cavities could reduce the skin friction drag [4].

For drag reduction techniques applied on an airfoil, using synthetic jets is an effective method of flow control that could reduce drag on a NACA 0015 airfoil [5]. The flow control technique using suction and blowing over a NACA 0012 airfoil could improve its aerodynamic characteristics [6]. The use of riblets is a useful method for the flow control and improves aerodynamic characteristics of a NACA 4415 airfoil [7]. The use of surface contour bumps of an airfoil could reduce the transonic drag [8].

To reduce drag on the Ahmed body, flaps could be used on the rear end of the body [9]. The use of an unsteady jet on the separation edges on the rear part of the body could reduce drag [10]. By forcing large-scale coherent streaks on the roof of the body, drag could be reduced [11]. The use of a continuous suction could reduce drag on a simplified car body [12]. The wake flow control technique by means of a synthetic jet could also reduce drag [13]. All the flow control techniques applied on these simplified models are described in details in the scientific literature.

In the present work, three cases have been studied. For these cases, different and simple configurations have been tested and which to our knowledge have not been treated before. The first case concerns the impact of the 
corrugations (directions and orientations) on the airflow over a flat plate. The drag reduction is obtained for a specific shape of the plate. These results found could be used at the level of habitats or mobile vehicles. The second classic case presents the impact of a drag reduction device on the aerodynamic performance of the NACA 0012 airfoil. For a specific orientation of this device, lift to drag ratio could be increased. The last case consists in replacing the jet flow control methods studied by several authors by taking the flow upstream of the Ahmed body. This simple and original device reduces drag and does not require an external supply of energy.

\section{Numerical modeling}

For all the studied cases, we consider an incompressible viscous flow in the steady state. For the case of laminar flows, Navier-stokes equations are defined by:

$$
(\mathbf{U} . \nabla) \mathrm{U}=\nu \nabla^{2} \mathrm{U}-\frac{1}{\rho} \nabla p
$$

where $v$ is the kinematic viscosity, $\rho$ is the density, $p$ is the pressure and $\mathbf{U}(u, v, w)$ is the velocity vector.

For turbulent flows, Reynolds-averaged Navier-Stokes (RANS) equations are defined by:

$$
(\mathbf{U} . \nabla) \mathrm{U}=\nu \nabla^{2} \mathrm{U}-\frac{1}{\rho} \nabla p+\nabla \tau .
$$

In these equations, $\mathbf{U}(u, v, w)$ represents the mean velocity vector, while $\tau$ represents the Reynolds stresses.

The velocity fluctuations are not available in RANS simulations. For that reason, closure models must be used in order to provide the Reynolds stresses.

In this study, the SST $k-\omega$ turbulent model is used to capture the effect of turbulent flow conditions. More information about this model is found in literature [14].

The Reynolds number is given by:

$$
\operatorname{Re}=\frac{U L}{v}
$$

where $U$ is the freestream velocity and $L$ is the characteristic length.

The drag coefficient $C_{d}$ and the lift coefficient $C_{l}$ are defined by:

$$
\begin{gathered}
C_{d}=\frac{F_{d}}{\frac{1}{2} \rho U^{2} A} \\
C_{l}=\frac{F_{l}}{\frac{1}{2} \rho U^{2} A}
\end{gathered}
$$

where $F_{d}$ is the drag force, $F_{l}$ is the lift force and $A$ is the reference area.

The lift to drag ratio is used to measure the efficiency of an airfoil. It is defined by:

$$
\text { Ratio }=\frac{F_{l}}{F_{d}}=\frac{C_{l}}{C_{d}} .
$$

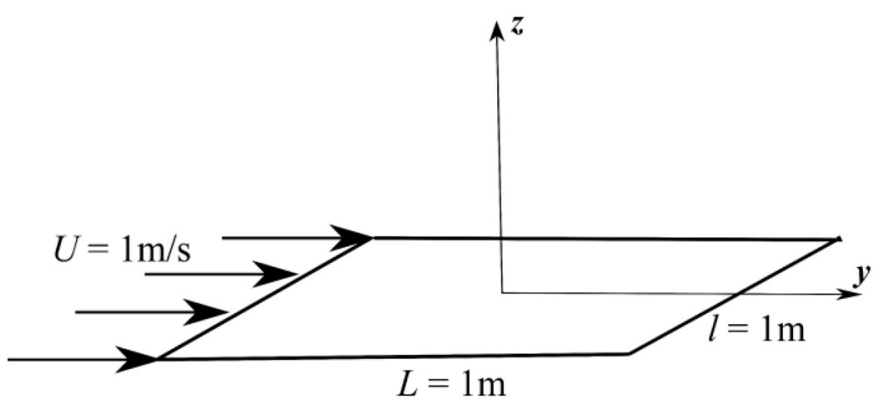

Fig. 1. Flat plate dimensions.

\section{Description of the different test cases}

In this section, we present the description of the different techniques proposed to reduce drag on the simplified models. These techniques are tested on a flat plate, a NACA 0012 airfoil and a simplified vehicle model (Ahmed body). All simulations have been performed using the CFD software Ansys Fluent 17.0.

\subsection{Flat plate}

Several fields (construction, automobile, etc.) could benefit from the use of corrugations of flat plates. In fact, corrugations could reduce drag on flat plates for low Reynolds numbers.

We study the effect of corrugating the shape of a flat plate on the drag reduction in the case of laminar flows [15]. Numerical simulations on various shapes and orientations of the corrugations have been performed for low Reynolds numbers.

We consider a tridimensional flow over a flat plate (reference plate) with $1 \mathrm{~m}$ of length and $1 \mathrm{~m}$ of width. Figure 1 shows the flat plate dimensions.

We modify the shape of the plate. Figure 2 shows the different shapes of the flat plate. They have the same dimensions of the reference plate $(1 \mathrm{~m} \times 1 \mathrm{~m})$ and they are tested by the same flow conditions. The corrugated plate (Fig. 2b) and the inversed corrugated plate (Fig. 2c) are identical but they are exposed to airflow with different directions. Similarly, for the convergent corrugated plate (Fig. 2d) and the divergent corrugated plate (Fig. 2e). The same for the sinusoidal plate (Fig. 2f) and the inverted sinusoidal plate (Fig. $2 \mathrm{~g}$ ).

The boundary conditions of our simulations are represented in Figure 3. These conditions are detailed in Figure 4 . The air has an inlet velocity of $1 \mathrm{~m} / \mathrm{s}$, a density of $1.225 \mathrm{~kg} / \mathrm{m}^{3}$ and a viscosity of $1.7894 \mathrm{e}^{-05} \mathrm{~kg} / \mathrm{m}$.s.

All tests have been performed for low Reynolds numbers $(\mathrm{Re}=10, \mathrm{Re}=50, \mathrm{Re}=100, \mathrm{Re}=500$ and $\mathrm{Re}=$ 1000). The laminar model has been used.

\subsection{NACA 0012 airfoil}

We present the effect of using the drag reduction device on the aerodynamic performances of the NACA 0012 airfoil.

At first, we study the separation around the NACA 0012 airfoil shown in Figure 5 (Chord length $c=1 \mathrm{~m}$ ) for an 


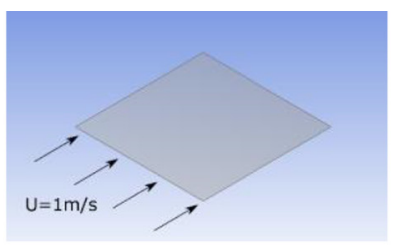

(a)

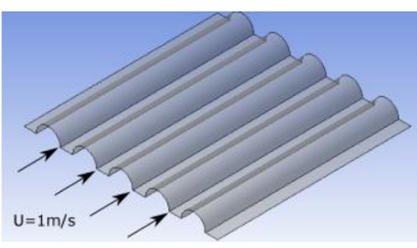

(b)

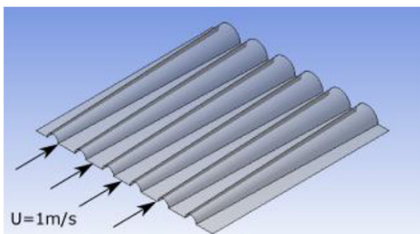

(d)

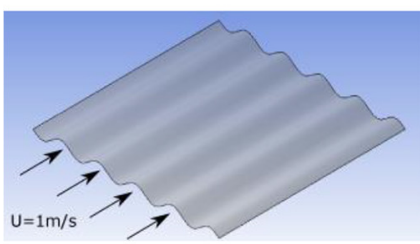

(f)

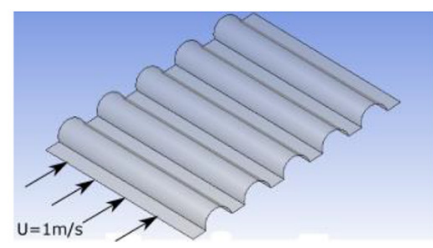

(c)

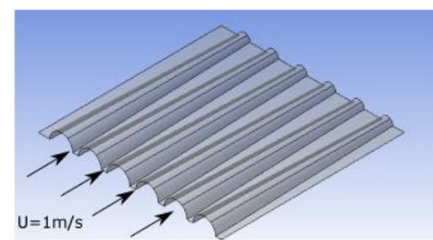

(e)

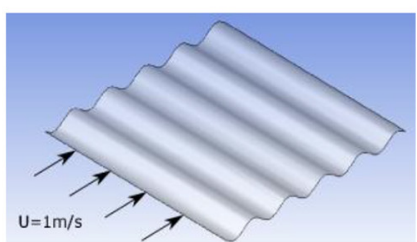

(g)
Fig. 2. The different shapes of the plate (a) Reference plate (b) Corrugated plate (c) Inverted corrugated plate (d) Convergent corrugated plate (e) Divergent corrugated plate (f) Sinusoidal plate (g) Inverted sinusoidal plate.

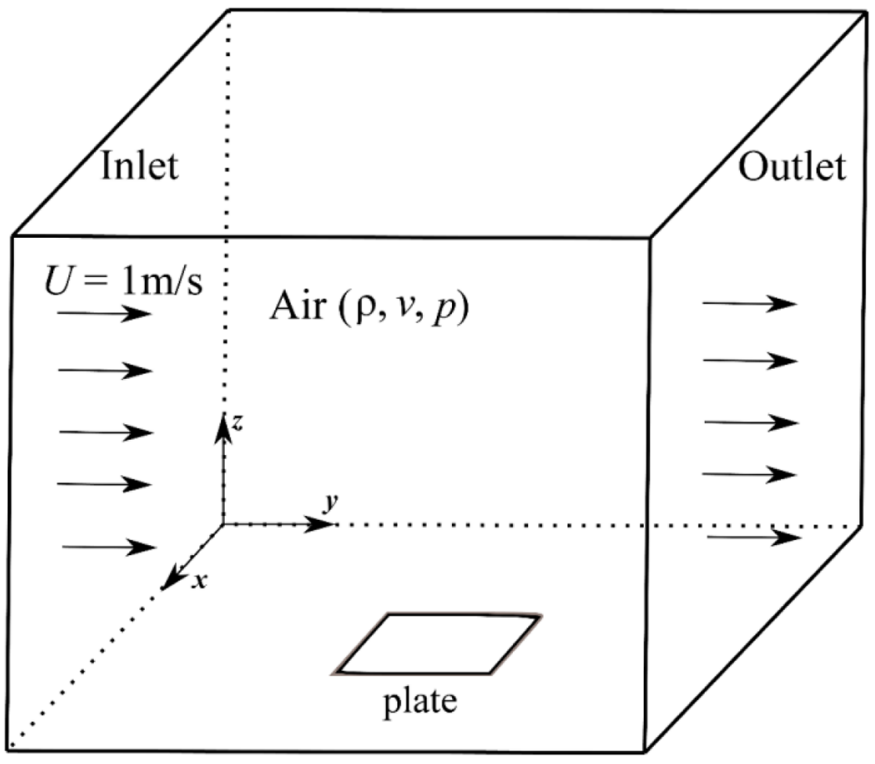

Fig. 3. Boundary conditions of numerical simulations.

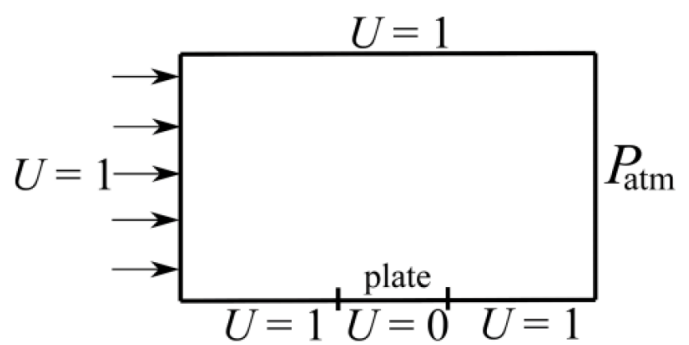

(a)

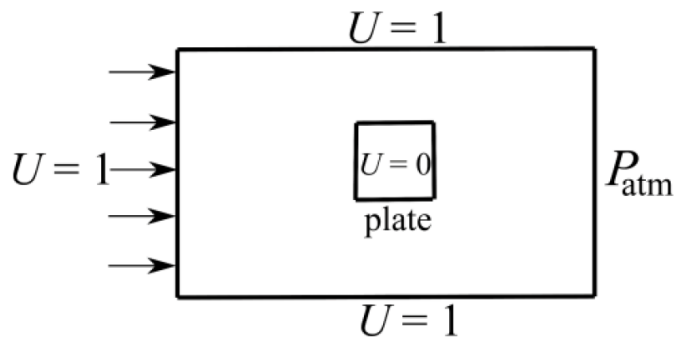

(b)

Fig. 4. Description of the boundary conditions.

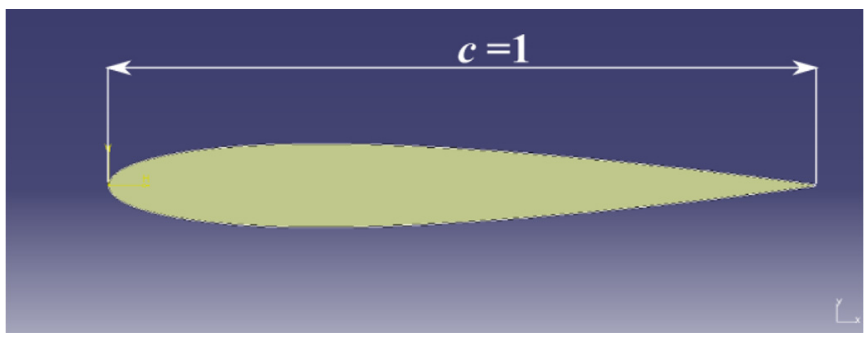

Fig. 5. NACA 0012 airfoil.

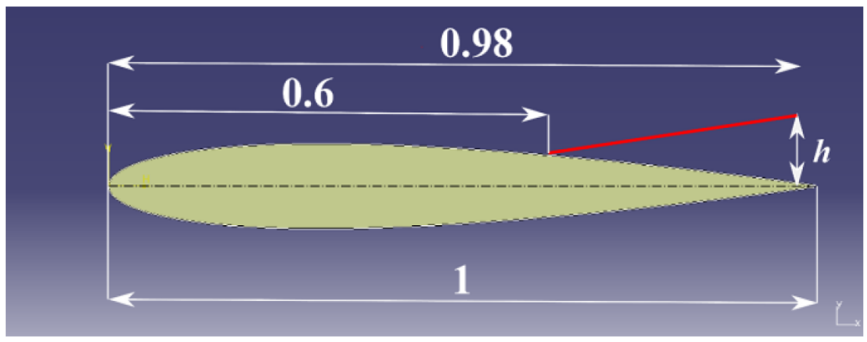

Fig. 6. Installation of the drag reduction device on the NACA 0012 airfoil for the angle of attack $5^{\circ}$.

incompressible flow for an angle of attack of $5^{\circ}$. Numerical simulations have been performed in the two-dimensional case.

The proposed device is fixed at the point of the flow separation on the airfoil. The flow separation occurs at the distance $0.6 \mathrm{~m}$ from leading edge as shown in Figure 6 . Several configurations have been evaluated by modifying the length $h$ which characterizes the orientation of the device.

The boundary conditions of our numerical simulations are detailed in Figure 7. $\alpha$ represents the angle of attack of 


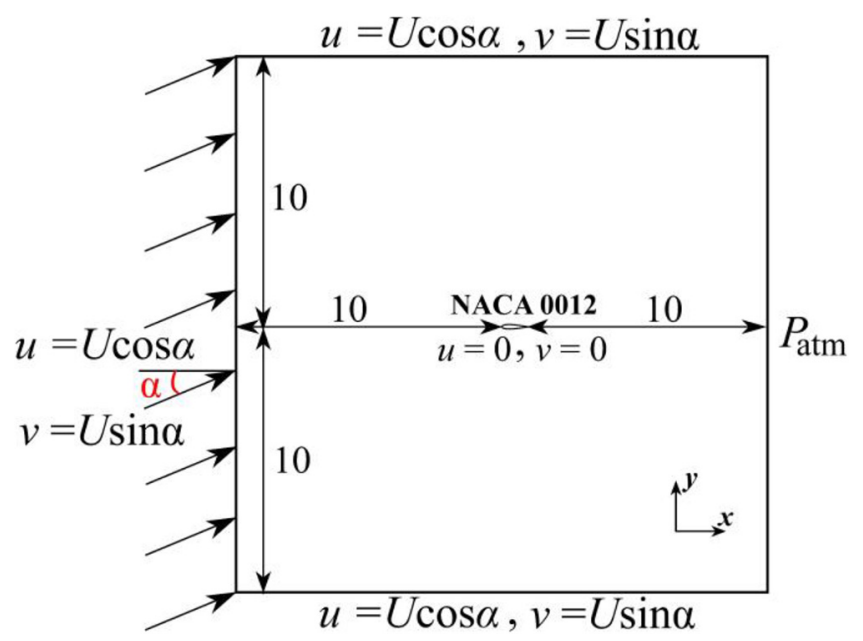

Fig. 7. Boundary conditions for numerical simulations on the NACA 0012 airfoil.

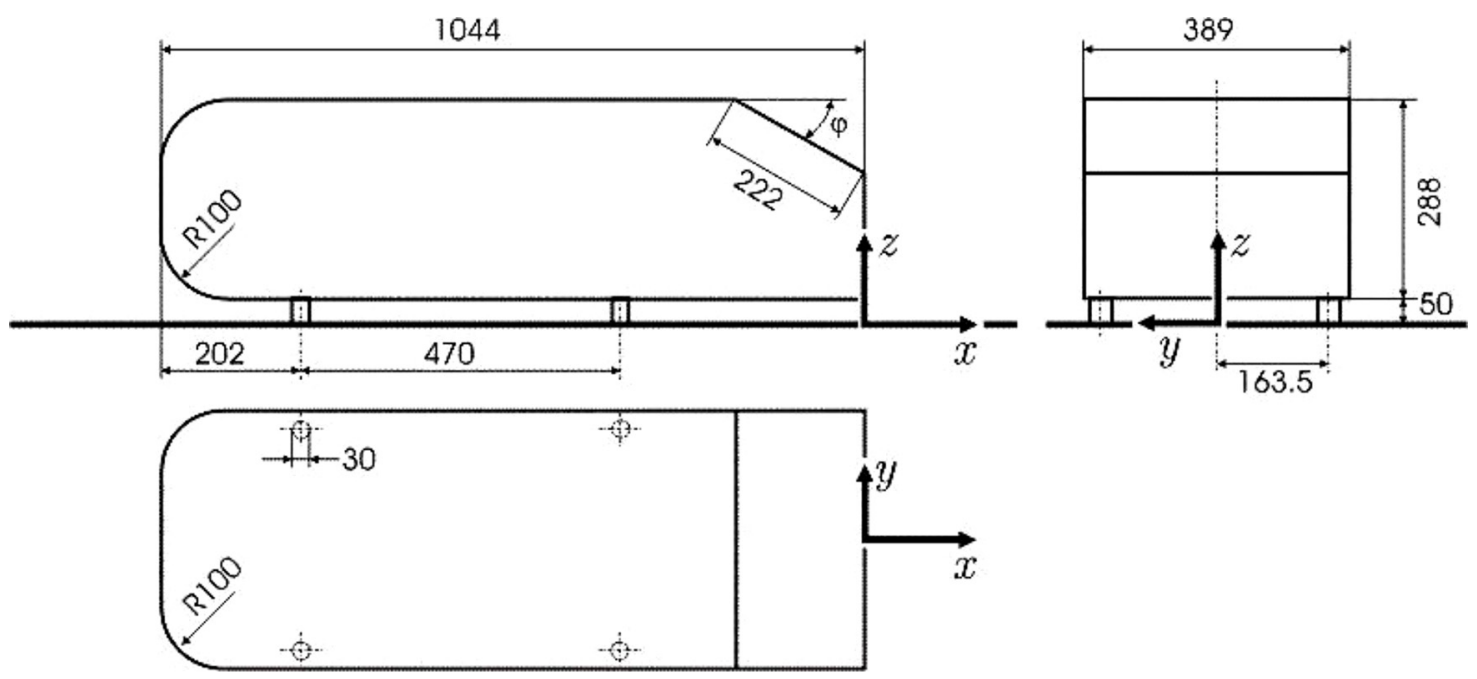

Fig. 8. The Ahmed body geometry.

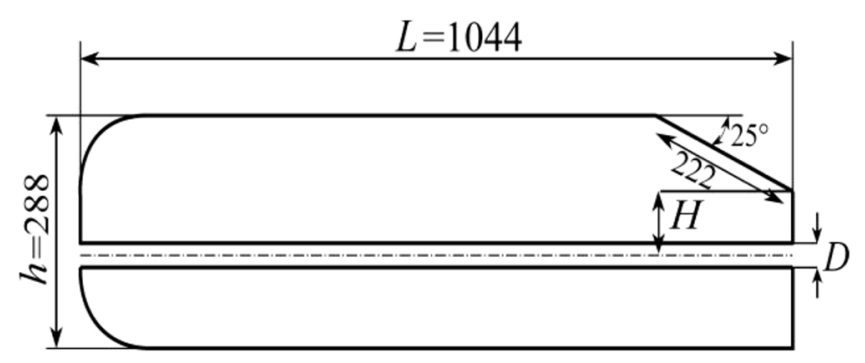

Fig. 9. Characteristics of the conduit.

the airfoil and $U$ is the air velocity at the inlet $(U=1 \mathrm{~m} / \mathrm{s})$. The velocity components depend on the angle of attack $\alpha \quad(u=U \cos \alpha, v=U \sin \alpha)$. The air has a density of $1.225 \mathrm{~kg} / \mathrm{m}^{3}$ and a viscosity of $1.7894 \mathrm{e}^{-05} \mathrm{~kg} / \mathrm{m}$.s.

Numerical simulations have been performed for a Reynolds number $\mathrm{Re}=1000$. The laminar model has been used.

\subsection{Ahmed body}

We study an original and simple technique used to reduce the drag on the Ahmed body without using external supplies of energy.

This technique consists of perforating a conduit on the Ahmed body. The role of this conduit is leading a part from the incoming flow to be injected in the recirculation zone in the rear end of the body. Numerical simulations have been performed in the two-dimensional case.

Figure 8 represents the geometry of the Ahmed body. Dimensions are in $\mathrm{mm}$ in Figure 8 till Figure 12.

We perforate a conduit in the body as shown in Figure $9 . D$ is the diameter of the conduit and $H$ is its position from the lower edge of the slant.

To test the effect of decreasing the conduit diameter at the exit. The conduit diameter is reduced at the outlet as shown in Figure 10. $d$ is the conduit diameter at the exit. 


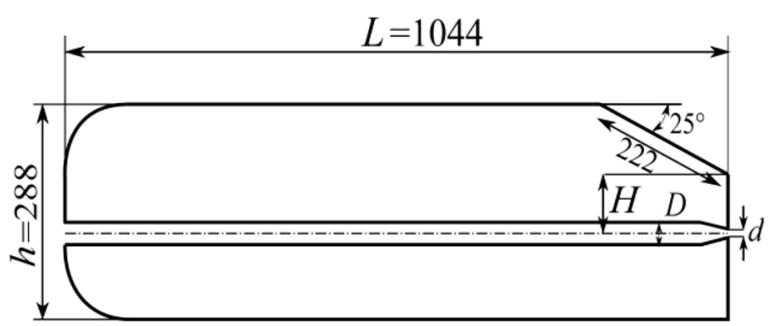

(a)

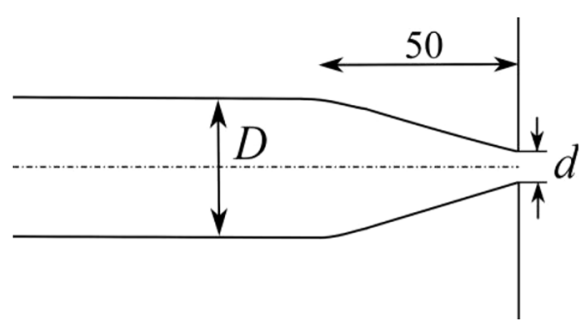

(b)

Fig. 10. Narrowing the conduit exit (a) General view (b) Zoom on the conduit exit.

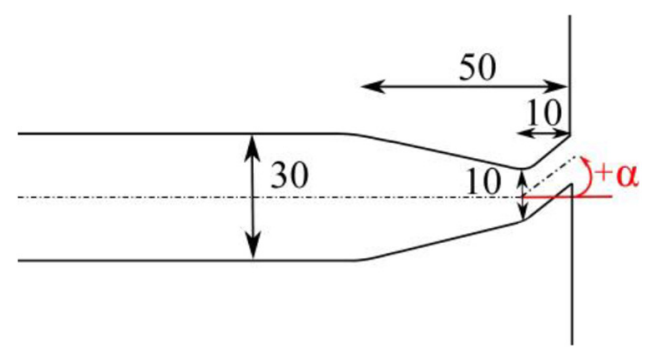

Fig. 11. Inclination of the conduit at the outlet.

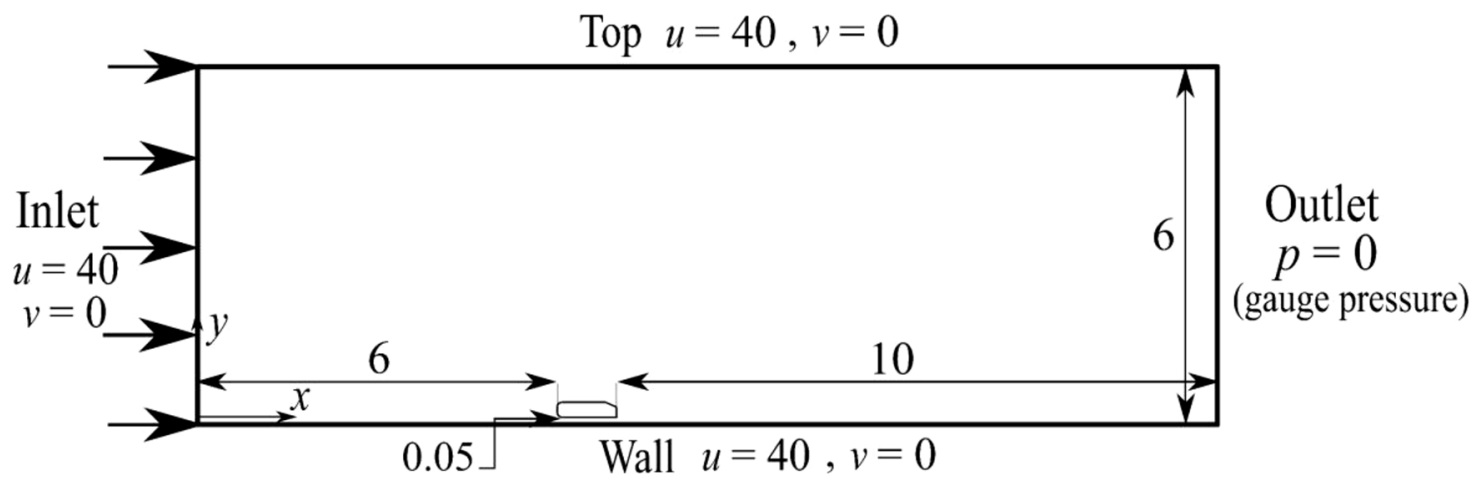

Fig. 12. Boundary conditions for simulations on the Ahmed body.

The inclination of the conduit exit is also tested. $\alpha$ represents the angle of inclination as shown in Figure 11.

The boundary conditions of our simulations on the Ahmed body are presented in Figure 12. The air has an inlet velocity of $40 \mathrm{~m} / \mathrm{s}$, a density of $1.225 \mathrm{~kg} / \mathrm{m}^{3}$ and a viscosity of $1.7894 \mathrm{e}^{-05} \mathrm{~kg} / \mathrm{m}$.s.

All tests have been performed for a Reynolds number $\mathrm{Re}=2.85 \mathrm{e} 06$. The SST $k-\omega$ turbulent model has been used.

For the three cases treated in this study, various tests have been carried out in order to prove the precision of our numerical calculations.

To avoid redundancy and for the clarity of this article, we present only the study of the grid influence on the numerical calculations in the case of flow on the Ahmed body without using the new device.

Table 1 certifies that the drag coefficient of the numerical simulations for the case of the Ahmed body is grid independent. To ensure the accuracy of the simulations. The grid of 60285 elements is used in the next simulations.
Table 1. The grid independence study for the case of the Ahmed body.

\begin{tabular}{ll}
\hline Number of mesh elements & $C_{d}$ \\
\hline 30383 & 0.3024 \\
42706 & 0.3015 \\
52628 & 0.3008 \\
60285 & 0.3008 \\
62137 & 0.3008 \\
\hline
\end{tabular}

\section{Results and discussions}

In this section, we present the different results obtained in our numerical simulations. Drag coefficients of our models are compared before and after using the drag reduction techniques. For the NACA 0012 airfoil, the lift to drag ratio is also used for comparison. 
Table 2. Drag coefficient of the reference plate compared to corrugated and inverted corrugated plates.

\begin{tabular}{|c|c|c|c|c|c|}
\hline \multirow[t]{2}{*}{ Re } & \multirow{2}{*}{$\frac{\text { Reference plate }}{C_{d}}$} & \multicolumn{2}{|c|}{ Corrugated plates } & \multicolumn{2}{|c|}{ Inverted corrugated plates } \\
\hline & & $C_{d}$ & $\frac{\Delta C_{d}}{C_{d}}$ & $C_{d}$ & $\frac{\Delta C_{d}}{C_{d}}$ \\
\hline 10 & 2.305 & 3.048 & $32.2 \%$ & 4.002 & $73.6 \%$ \\
\hline 50 & 0.562 & 0.725 & $33.8 \%$ & 0.996 & $77.2 \%$ \\
\hline 100 & 0.325 & 0.415 & $27.7 \%$ & 0.609 & $87.4 \%$ \\
\hline 500 & 0.105 & 0.132 & $25.7 \%$ & 0.248 & $136.2 \%$ \\
\hline 1000 & 0.068 & 0.085 & $25 \%$ & 0.182 & $167.6 \%$ \\
\hline
\end{tabular}

Table 3. Drag coefficient of the reference plate compared to convergent corrugated and divergent corrugated plates.

\begin{tabular}{|c|c|c|c|c|c|}
\hline \multirow[t]{2}{*}{$\operatorname{Re}$} & \multirow{2}{*}{$\frac{\text { Reference plate }}{C_{d}}$} & \multicolumn{2}{|c|}{ Convergent corrugated plates } & \multicolumn{2}{|c|}{ Divergent corrugated plates } \\
\hline & & $C_{d}$ & $\frac{\Delta C_{d}}{C_{d}}$ & $C_{d}$ & $\frac{\Delta C_{d}}{C_{d}}$ \\
\hline 10 & 2.305 & 3.294 & $42.9 \%$ & 3.391 & $47.2 \%$ \\
\hline 50 & 0.562 & 0.780 & $38.8 \%$ & 0.776 & $38.1 \%$ \\
\hline 100 & 0.325 & 0.445 & $36.9 \%$ & 0.432 & $32.9 \%$ \\
\hline 500 & 0.105 & 0.141 & $34.3 \%$ & 0.127 & $21 \%$ \\
\hline 1000 & 0.068 & 0.092 & $35.3 \%$ & 0.079 & $11 \%$ \\
\hline
\end{tabular}

Table 4. Drag coefficient of the reference plate compared to sinusoidal and inverted sinusoidal plates.

\begin{tabular}{|c|c|c|c|c|c|}
\hline \multirow[t]{2}{*}{ Re } & \multirow{2}{*}{$\frac{\text { Reference plate }}{C_{d}}$} & \multicolumn{2}{|c|}{ Sinusoidal plate } & \multicolumn{2}{|c|}{ Inverted sinusoidal plates } \\
\hline & & $C_{d}$ & $\frac{\Delta C_{d}}{C_{d}}$ & $C_{d}$ & $\frac{\Delta C_{d}}{C_{d}}$ \\
\hline 10 & 2.305 & 2.977 & $29.2 \%$ & 2.071 & $-10.2 \%$ \\
\hline 50 & 0.562 & 0.703 & $25.1 \%$ & 0.527 & $-6.2 \%$ \\
\hline 100 & 0.325 & 0.399 & $22.7 \%$ & 0.314 & $-3.4 \%$ \\
\hline 500 & 0.105 & 0.123 & $17.1 \%$ & 0.107 & $1.9 \%$ \\
\hline 1000 & 0.068 & 0.078 & $14.7 \%$ & 0.070 & $2.9 \%$ \\
\hline
\end{tabular}

\subsection{Effects of corrugations on flat plate drag}

To evaluate the effect of corrugations of the flat plate on the drag reduction, drag coefficients of the flat plate (Reference plate) and the different shapes of the plate (Fig. 2) are compared. In Tables $2-4$, we represent the drag coefficients of the different shapes as well as the relative drag modifications versus the reference plate for the various Reynolds numbers.

From the results obtained, the drag coefficient is inversely proportional to the Reynolds number for all the plate configurations. This result is in agreement with several formulas existing in the literature which express $C_{d}$ as a function of the Re.

For the Reynolds numbers studied, the inverted corrugated plate presents the most disapproving configuration. It dramatically increases $C_{d}$ of the plane plate. Such configurations should be avoided especially in low Reynolds number designs.
For the Reynolds numbers studied, the corrugated, the convergent corrugated and the divergent corrugated plates always increase the drag coefficient with respect to the flat plate.

The sinusoidal plate configuration has the lowest $C_{d}$ of all configurations, but its $C_{d}$ remains higher than the $C_{d}$ of the flat plate.

Finally, the inverted sinusoidal plate is the configuration that reduces the drag coefficient of the flat plate: This configuration reduces the drag coefficient by $10.2 \%$ for $\mathrm{Re}=10$, by $6.2 \%$ for $\mathrm{Re}=50$ and by $3.4 \%$ for $\mathrm{Re}=500$. This shape is recommended especially for low Reynolds numbers.

\subsection{Effects of the NACA 0012 drag reduction device}

Firstly, we calculate the aerodynamic coefficients for the flow over a NACA 0012 airfoil, for different angles of attack and for the Reynolds number $\mathrm{Re}=1000$. We compare these 


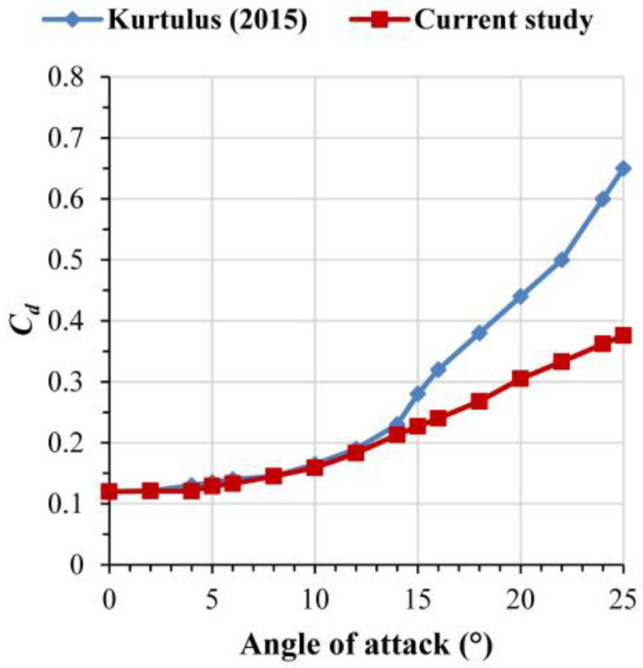

(a)

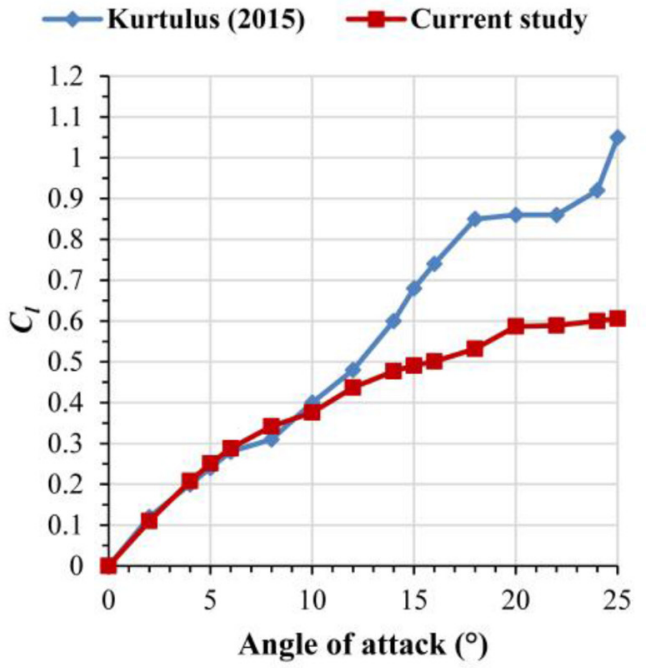

(b)

Fig. 13. Comparison with results obtained by (Kurtulus, 2015) (a) of the drag coefficients and (b) of the lift coefficients obtained in the present study for different angles of attack of the NACA 0012 airfoil.

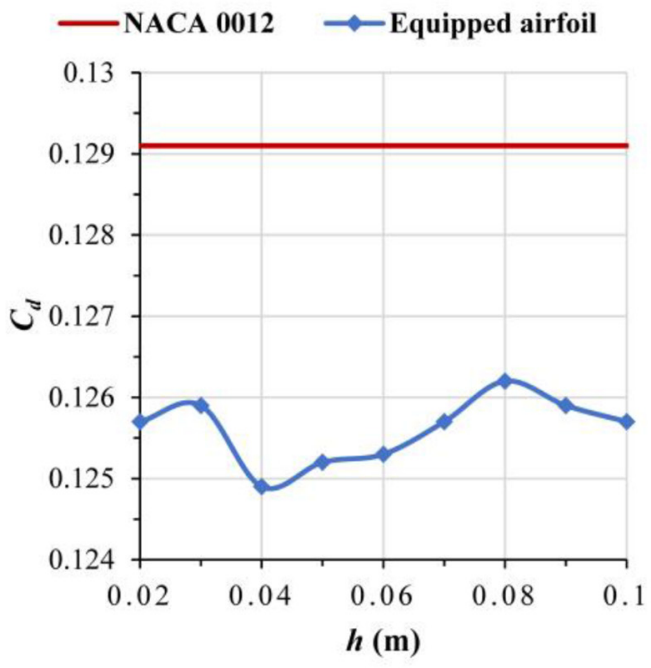

(a)

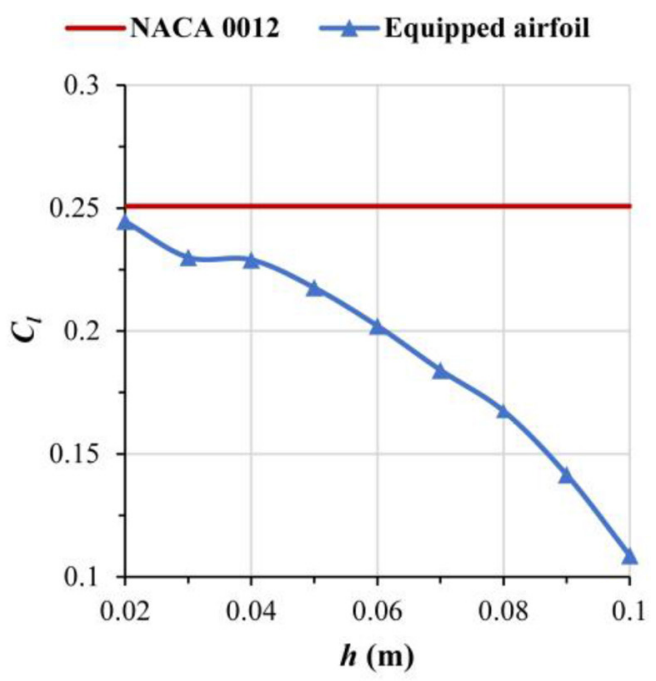

(b)

Fig. 14. (a) Drag coefficient evolution with variation of the height $h$ of the device (b) Lift coefficient evolution with variation of the height $h$ of the device.

results with those obtained in literature [16]. Figure 13 shows that from the angle of attack $\alpha=0^{\circ}$ till $14^{\circ}$, the drag and lift coefficients found in our simulations are in good agreement with results obtained in literature.

Starting from $\alpha=15^{\circ}$, the values of the two aerodynamic coefficients begin to differ from the values found in previous works. This is probably due to the flow instabilities at these angles of attack [16]. There are even authors who have stopped the calculations of the two coefficients at the angle of attack $15^{\circ}$ [17], other authors have stopped calculations at the angle of attack $18^{\circ}$ [18].

In the rest of this study, the angle of attack $5^{\circ}$ is chosen.
We compare the aerodynamic coefficients of the NACA 0012 airfoil (without device) with those of the different configurations of the airfoil equipped with the drag reduction device. Each configuration is characterized by a modification of the height $h$ which characterizes the orientation of the device. Figure 14 illustrates the evolution in drag and lift coefficients with the variation in the height $h$ for a Reynolds number $\mathrm{Re}=1000$.

Table 5 represents the drag and lift coefficients as well as the relative drag modifications versus the reference airfoil (NACA 0012 without using the device) for the different configurations. 
Table 5. Drag and lift coefficients obtained for the different values of the height $h$.

\begin{tabular}{llllll}
\hline$h(\mathrm{~m})$ & $C_{d}$ & $\frac{\Delta C_{d}}{C_{d}}$ & $C_{l}$ & $\frac{\Delta C_{l}}{C_{l}}$ & $\frac{C_{l}}{C_{d}}$ \\
\hline Without device & 0.1291 & 0 & 0.2508 & 0 & 1.9427 \\
0.02 & 0.1257 & $-2.63 \%$ & 0.2446 & $-2.47 \%$ & 1.9460 \\
0.03 & 0.1259 & $-2.48 \%$ & 0.2300 & $-8.29 \%$ & 1.8268 \\
0.04 & 0.1249 & $-3.25 \%$ & 0.2290 & $-8.69 \%$ & 1.8335 \\
0.05 & 0.1252 & $-3.02 \%$ & 0.2176 & $-13.24 \%$ & 1.7380 \\
0.06 & 0.1253 & $-2.94 \%$ & 0.2021 & $-19.27 \%$ & 1.8563 \\
0.07 & 0.1257 & $-2.63 \%$ & 0.1841 & $-26.59 \%$ & 1.4646 \\
0.08 & 0.1262 & $-2.25 \%$ & 0.1675 & $-33.21 \%$ & 1.3273 \\
0.09 & 0.1259 & $-2.48 \%$ & 0.1416 & $-43.54 \%$ & 1.1247 \\
0.1 & 0.1257 & $-2.63 \%$ & 0.1087 & $-56.66 \%$ & 0.8648 \\
\hline
\end{tabular}

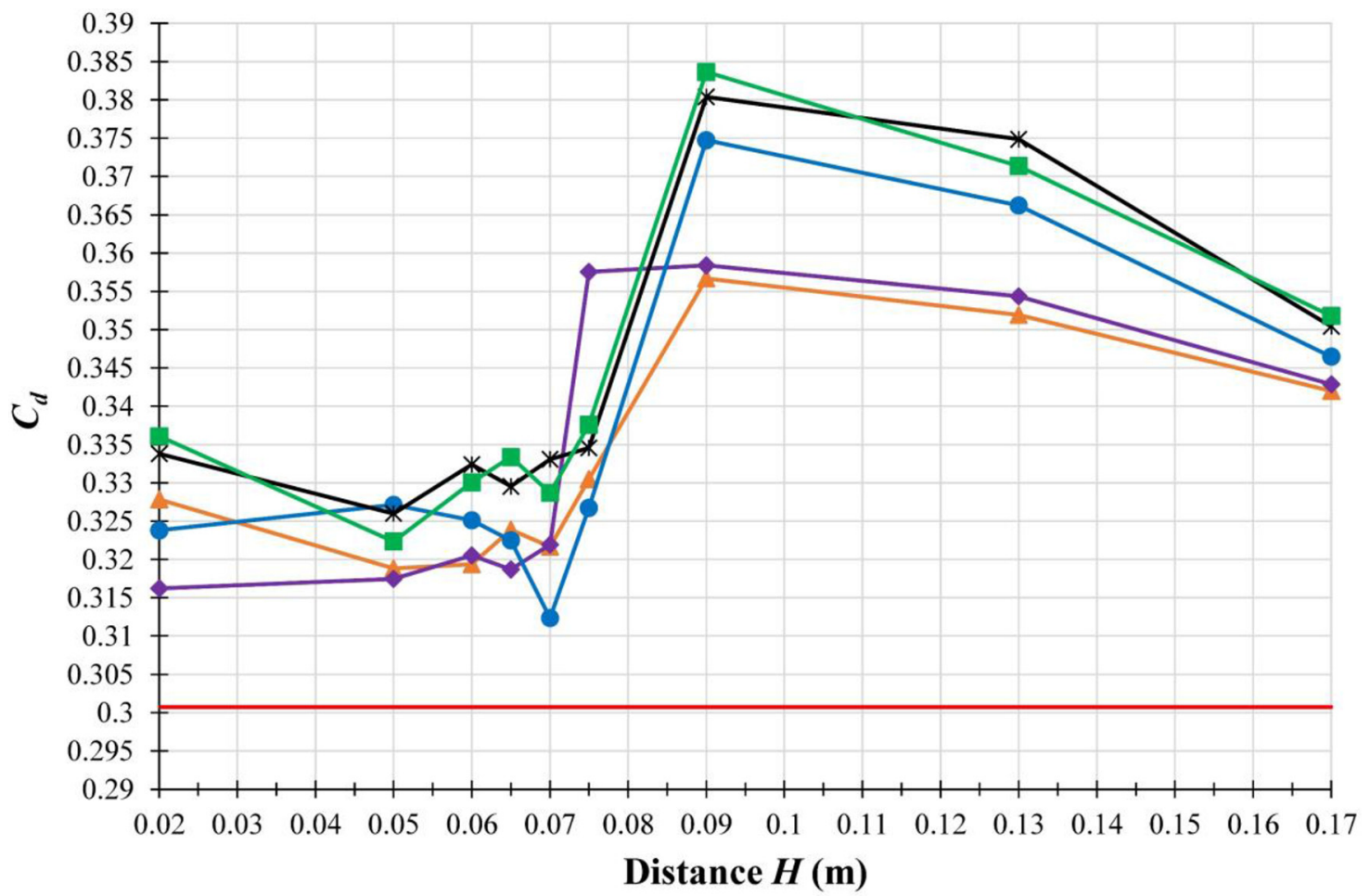

$\longrightarrow$ Ahmed body $\rightarrow-\mathrm{D}=0.01 \mathrm{~m} \rightarrow \mathrm{D}=0.015 \mathrm{~m} \rightarrow \mathrm{D}=0.02 \mathrm{~m} \rightarrow \mathrm{D}=0.025 \mathrm{~m}-\mathrm{D}=0.03 \mathrm{~m}$

Fig. 15. Drag evolution with the distance from the lower slant edge for various conduit diameters.

For all values of the height $h$ included between $0.02 \mathrm{~m}$ and $0.1 \mathrm{~m}$, the drag coefficient decreases compared to the drag coefficient of the reference airfoil with a maximum decrease of $3.25 \%$ for a value of $h=0.04 \mathrm{~m}$. On the other hand, a decrease in the coefficient of lift is also noticed for all the values of $h$, with a large decrease starting from $h=0.07 \mathrm{~m}$.

For all configurations of the device, the drag and lift coefficients decrease. Consequently, a decrease in the ratio $\frac{C_{l}}{C_{d}}$ compared to the reference airfoil is remarked.

Only the first configuration (for the value of $h=0.02 \mathrm{~m}$ ) makes it possible to reduce the drag while increasing the lift to drag ratio $\frac{C_{l}}{C_{d}}$.

\subsection{Effects of the conduit on the Ahmed body drag}

For different configurations of the conduit, the reference drag coefficient (of the body without using the conduit) is compared with those of the body using the conduit.

First tests are performed using conduits with various diameters $D$. Different positions of the conduit characterized by the distance $H$ from the lower edge of the Ahmed body slant have been tested. Figure 15 shows the evolution of the drag coefficient for various diameters of the conduit placed in various distances from the lower edge. 


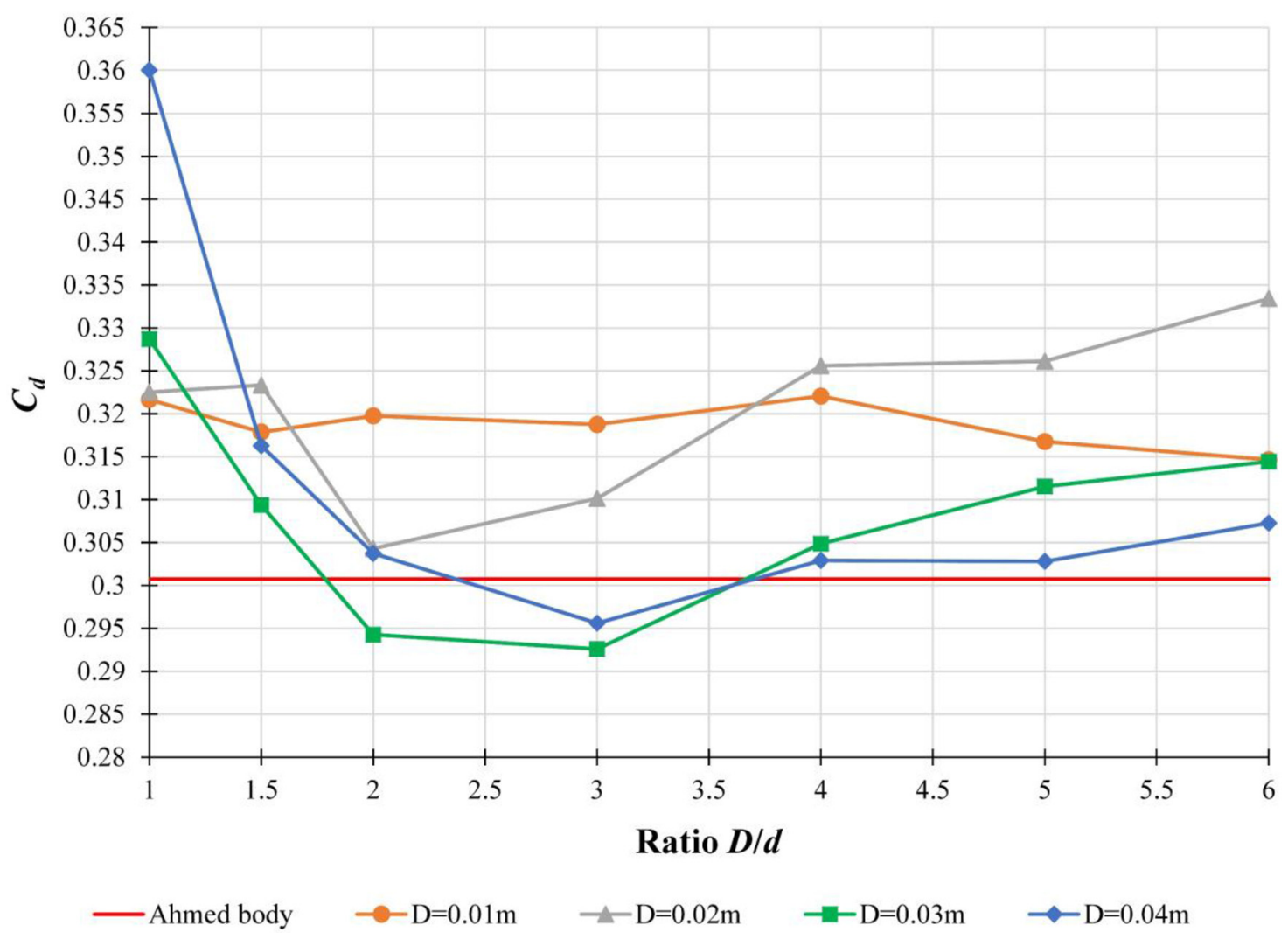

Fig. 16. Drag evolution with the different Ratios $D / d$ for different conduit diameters.

For all the conduit configurations, drag coefficients are higher than the reference drag coefficient. Most configurations present lower drag coefficient at the distance $H=0.07 \mathrm{~m}$. For that reason, this distance is chosen in the next simulations.

Second tests are performed using a conduit which is narrowed at the outlet. Figure 16 shows the evolution of drag coefficient with different ratios $D / d$ (diameter of the conduit/ diameter at the exit).

For $D / d=3$ and the conduit of diameter $D=0.03 \mathrm{~m}$, the drag coefficient is decreased by $2.73 \%$. This configuration is the chosen one, it has a maximum drag reduction.

In the next simulations, this suitable configuration $(D / d=3$ and $D=0.03 \mathrm{~m})$ is kept for the same distance $H=0.07 \mathrm{~m}$.

In the final tests, the effect of the inclination of the exit axis is evaluated. Figure 17 shows the evolution of the drag coefficient with various angles of inclination of the conduit axis at the exit.

For negative angles of inclination, drag coefficients are higher in comparison with the reference drag coefficient.

The lower drag coefficient is obtained for the angle of inclination $\alpha=5^{\circ}$. This suitable configuration allows a maximum drag reduction of $6.42 \%$.

Between the angles of inclination $\alpha=5^{\circ}$ and $\alpha=25^{\circ}$, the drag coefficient increases but stay lower than the reference drag coefficient.
From the angle of inclination $\alpha=25^{\circ}$, the drag coefficient increases and exceeds the reference drag coefficient.

\section{Conclusion}

In the present paper, new drag reduction techniques have been investigated for three simplified models. The first method consists of the modification of the shape of a flat plate. Results confirm that the inverted sinusoidal plate is the convenient configuration. It reduces the drag coefficient of the plate for low Reynolds numbers. The second method is performed by using a device fixed on the separate point of a NACA 0012 airfoil. For all configurations of the drag reduction device, drag coefficient of the airfoil is reduced. The lift to drag ratio is reduced for a single configuration of the device characterized by the height $h=0.02 \mathrm{~m}$. The last method is obtained by perforating a conduit on the Ahmed body. This conduit allows to bringing a part of the flow from the inlet and inject it in the recirculation zone at the back of body. The suitable configuration is characterized by a conduit of diameter $D=0.03 \mathrm{~m}$ and exit diameter $d=0.01 \mathrm{~m}$. This conduit is placed at the distance $H=0.07 \mathrm{~m}$ from the lower edge of the Ahmed body slant and inclined at the exit by $\alpha=5^{\circ}$. 


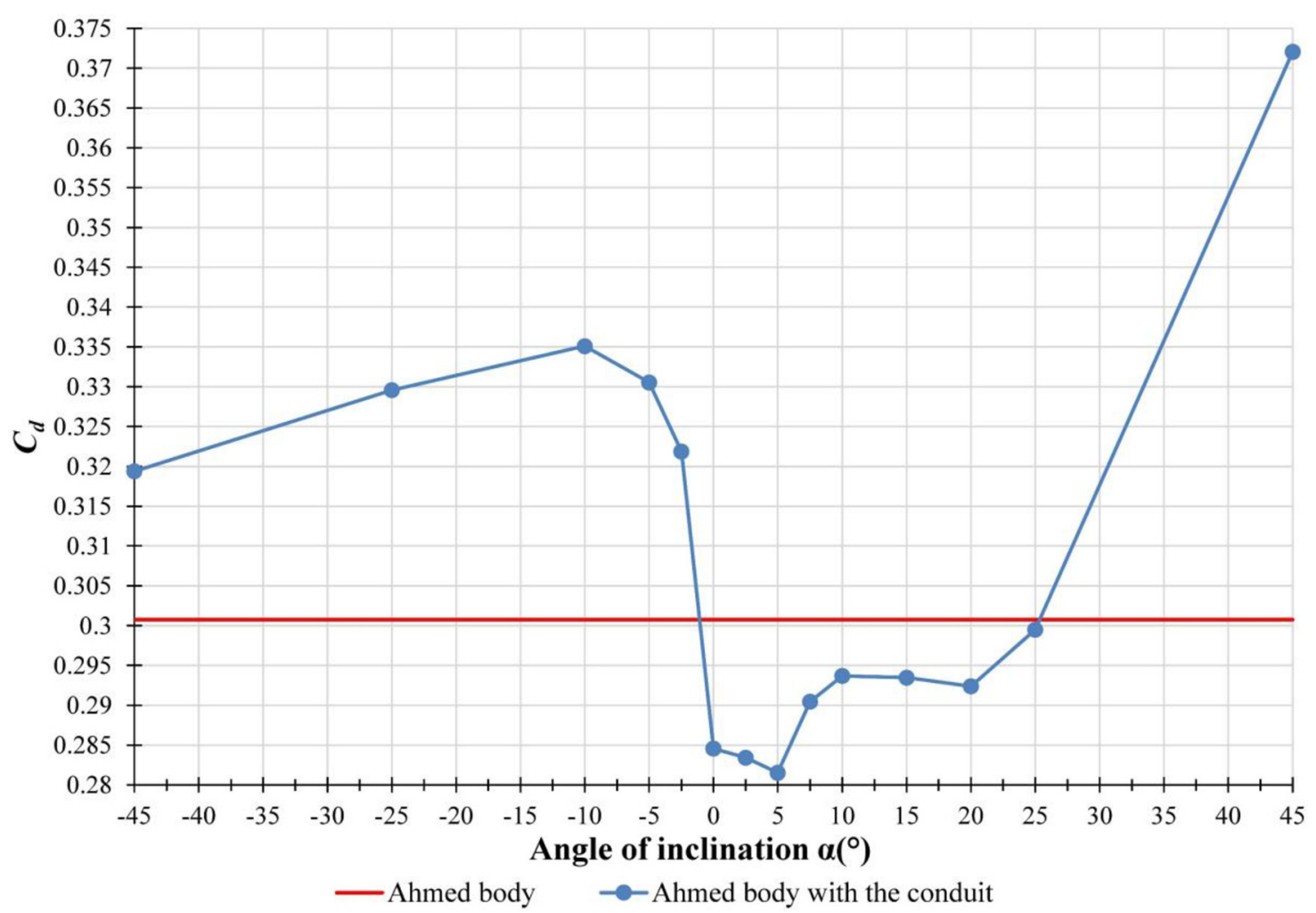

Fig. 17. Drag evolution with the angle of inclination of the outlet axis of the conduit.

Future works will focus on optimizing the shape of the flat plate to reduce drag in turbulent flows. A 3D study of the two devices used for the NACA 0012 airfoil and the Ahmed body will also be carried out. Finally, an experimental study is necessary in order to validate all these new drag reduction techniques.

\section{References}

1. W.C. Sanders, E.S. Winkel, D.R. Dowling, M. Perlin, S.L. Ceccio, Bubble friction drag reduction in a high-Reynoldsnumber flat-plate turbulent boundary layer, J. Fluid Mech. $\mathbf{5 5 2}, 353(2006)$

2. A. Heidarian, Numerical analysis of the effects of Riblets on drag reduction of a flat plate, J. Appl. Fluid Mech. 11, 679-688 (2018)

3. C. Chin, R. Örlü, P. Schlatter, J. Monty, N. Hutchins, Influence of a Large-Eddy-breakup-device on the turbulent interface of boundary layers, Flow Turbul. Combust. 99, 823-835 (2017)

4. E.R. Gowree, C. Jagadeesh, C.J. Atkin, Skin friction drag reduction over staggered three dimensional cavities, Aerosp. Sci. Technol. 84, 520-529 (2019)

5. D. You, P. Moin, Active control of flow separation over an airfoil using synthetic jets, J. Fluids Struct. 24, 1349-1357 (2008)
6. L. Huang, P.G. Huang, R.P. LeBeau, T. Hauser, Numerical study of blowing and suction control mechanism on NACA0012 airfoil, J. Aircr. 41, 1005-1013 (2004)

7. O.M. Fouatih, M. Medale, O. Imine, B. Imine, Design optimization of the aerodynamic passive flow control on NACA 4415 airfoil using vortex generators, Eur. J. Mech. B 56, 82-96 (2016)

8. W. Milholen, L. Owens, On the application of contour bumps for transonic drag reduction (invited), in $43 \mathrm{rd} A I A A$ Aerospace Sciences Meeting and Exhibit. American Institute of Aeronautics and Astronautics (2005)

9. J. Tian, Y. Zhang, H. Zhu, H. Xiao, Aerodynamic drag reduction and flow control of Ahmed body with flaps, Adv. Mech. Eng. 9, 168781401771139 (2017)

10. B. Wang, Z. Yang, H. Zhu, Active flow control on the $25^{\circ}$ Ahmed body using a new unsteady jet, Int. J. Heat Fluid Flow 79, 108459 (2019)

11. G. Pujals, S. Depardon, C. Cossu, Drag reduction of a 3D bluff body using coherent streamwise streaks, Exp. Fluids 49, 1085-1094 (2010)

12. M. Rouméas, P. Gilliéron, A. Kourta, Drag reduction by flow separation control on a car after body, Int. J. Numer. Meth. Fluids 60, 1222-1240 (2009)

13. A. Kourta, C. Leclerc, Characterization of synthetic jet actuation with application to Ahmed body wake, Sens. Actuators A: Phys. 192, 13-26 (2013)

14. F.R. Menter, Two-equation eddy-viscosity turbulence models for engineering applications, AIAA J. 32, 1598-1605 (1994) 
15. A. Agriss, M. Agouzoul, A. Ettaouil, Reducing drag on a flat plate subjected to incompressible laminar flow. Aniss S, editor. MATEC Web Conf. 286, 07006 (2019)

16. D.F. Kurtulus, On the Unsteady Behavior of the Flow around NACA 0012 Airfoil with Steady External Conditions at $\mathrm{Re}=1000$, Int. J. Micro Air Veh. 7, 301-326 (2015)

17. E.C. Douvi, D.P. Margaris, S.D. Lazaropoulos, S.G. Svanas, Experimental and computational study of the effects of different liquid water content on the aerodynamic performance of a Naca 0012 airfoil at Low Reynolds Number, in 5th International Conference on Experiments/ Process/System Modeling/Simulation/Optimization (2013), p. 9.

18. K. Yousefi, R. Saleh, P. Zahedi, Numerical study of blowing and suction slot geometry optimization on NACA 0012 airfoil, J. Mech. Sci. Technol. 28, 1297-1310 (2014)

Cite this article as: Amine Agriss, Mohamed Agouzoul, Abdeslem Ettaouil, Abdessamad Mehdari, Numerical study of new techniques drag reduction: application to aerodynamic devices, Int. J. Simul. Multidisci. Des. Optim. 12, 16 (2021) 